\title{
Scaling of X-ray Flux from High-Intensity Laser-Solid Interactions as a Function of Energy.
}

\author{
D. R. Rusby, C. M. Brenner, C. Armstrong, L. A. Wilson, R. Clarke, R. Deas, D. Lockley, S. Dorkings, K. \\ Butler, G. Cook, M. Carpenter, R.Giordmaina, A. Alejo, H. Ahmed, N. M. H. Butler, D. Haddock, A. \\ Higginson, A. McClymont, S. R. Mirfayzi, C. Murphy, M. Notley, P. Oliver, R. Allott, C. Hernandez-Gomez, \\ S. Kar, P. McKenna and D. Neely \\ Central Laser Facility, Rutherford Appleton Laboratory, Harwell Oxford, UK and Strathclyde University, Glasgow, UK \\ dean.rusby@stfc.ac.uk
}

Abstract: The bremsstrahlung x-rays from a laser-solid interaction have been investigated for the use of radiography. The scaling of the x-rays as a function of energy has been characterized and modelled and agrees with previous measurements.

\section{Introduction}

The $\mathrm{x}$-rays produced during a laser-solid interaction can be highly energetic $(>100 \mathrm{keV})$ and are typically emitted from a small region $(<100 \mu \mathrm{m})$. These attributes makes them favourable for x-ray radiography of dense materials. Many experiments have been conducted to characterize and optimize the $\mathrm{x}$-rays to produce the most suitable beams for radiography [1-3].

Here, the authors report on a scaling of the x-ray flux as a function of laser energy from an experimental campaign using a novel scintillator based detector. Modelling of the scaling of the $\mathrm{x}$-ray flux was conducted using the Monte Carlo code GEANT4 [4] and compared with results from the other sources.

\section{Experimental Results}

An experiment was conducted at the Vulcan laser [5] which achieves intensities up to $1.2 \times 10^{19} \mathrm{~W} / \mathrm{cm}^{2}$. The incident laser energy, $\mathrm{E}_{\mathrm{L}}$, was varied from 10 to $150 \mathrm{~J}$ in order to observe the scaling of the $\mathrm{x}$-ray flux from a $1 \mathrm{~mm} \mathrm{Al} \mathrm{target}$. The x-ray flux was measured using a scintillator array approximately $1 \mathrm{~m}$ away from the interaction position along the target normal. The scintillator array is designed such that the deeper layers detect higher energy $\mathrm{x}$-rays in comparison to the earlier layers. This allows the array to operate as an absorption spectrometer.

The average counts from the first 4 layers of the scintillator array, which are sensitive to $\mathrm{x}-$ rays from $50-500 \mathrm{keV}$, are shown in Figure 1. The flux of the $\mathrm{x}$-rays scales as $\mathrm{S}_{1 \rightarrow 4} \propto \mathrm{E}_{\mathrm{L}}{ }^{1.8 \rightarrow 2}$; which suggests the X-rays are becoming more energetic as a function of laser energy.

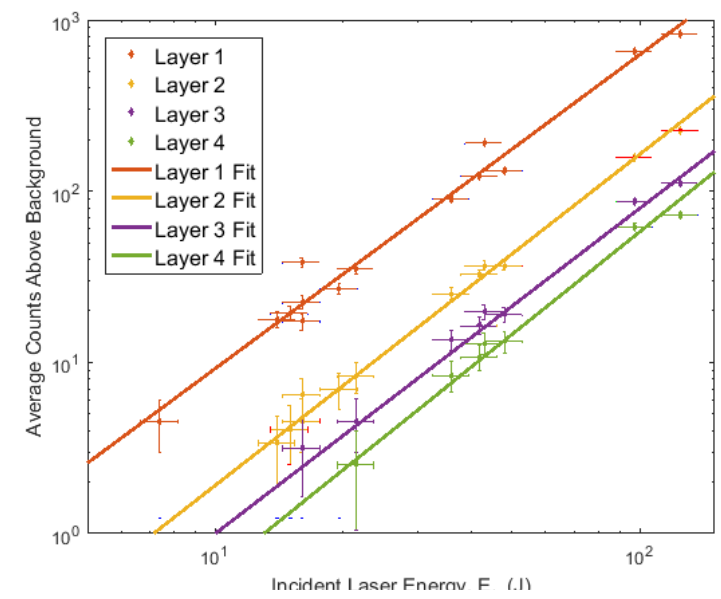

$$
\begin{aligned}
& S_{1} \propto E_{L}^{1.8 \pm 0.1} \\
& S_{2} \propto E_{L}^{1.9 \pm 0.1} \\
& S_{3} \propto E_{L}^{1.9 \pm 0.2} \\
& S_{4} \propto E_{L}^{2 \pm 0.2}
\end{aligned}
$$

Figure 1: Average counts from the scintillator array as a function of incident laser energy, $\mathrm{E}_{\mathrm{L}}$.

\section{Monte Carlo Modelling}

The modelling of the experimental results were conducted using the Monte Carlo Code GEANT4. The simulations were conducted using an electron beam containing $10^{6}$ particles with a relativistic-Maxwellian energy distribution | which were propagated through a $1 \mathrm{~mm}$ aluminum target. The simulation was repeated for a range of temperatures (200-1000keV). The emitted x-rays and electrons from each simulation were recorded using ideal detectors in-front and behind the target. The electrons that reach the rear of the target during laser-solid interactions are likely to 

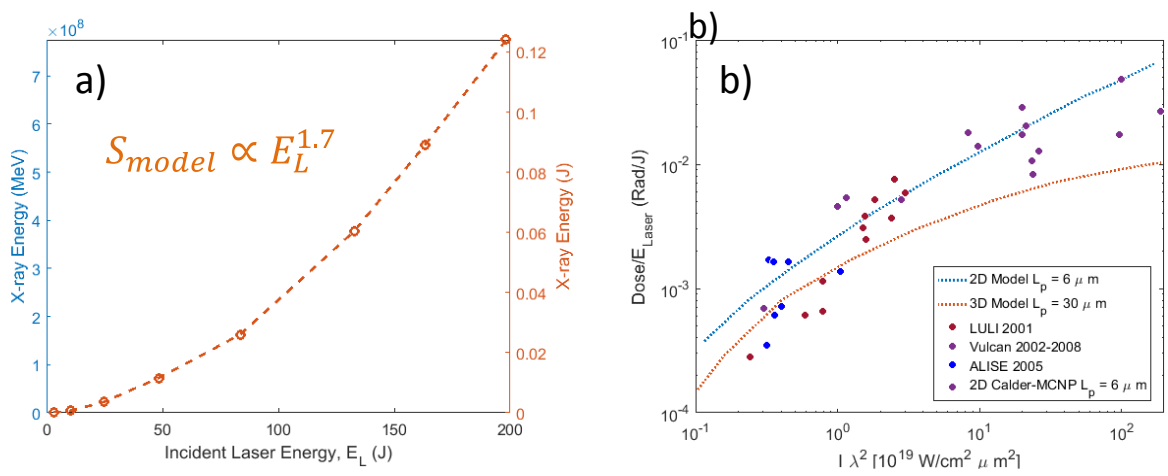

Figure 2: a) The results from the GEANT4 modelling to determine the scaling of $x$-ray flux as a function of incident laser energy. b) The $\mathrm{x}$-ray dose per incident laser energy as a function of intensity from numerical and analytical modelling by Fontaine et al [7].

undergo refluxing; this means that they are reflected back into the target due to the electro-static potential setup on the rear. To take this into account when considering the x-ray flux, the fraction of surviving electron fraction was calculated from the first pass. This fraction of the $\mathrm{x}$-ray energy was added to the $\mathrm{x}$-ray energy from one pass. This is repeated multiple times until the electron numbers have fallen below $5 \%$ of the original number.

The temperature of the input electrons was converted to an incident energy using the experimental parameters and using the Beg scaling [6] to convert temperature to intensity. The normalised x-ray flux from the simulations as a function of incident laser energy is shown in Figure 2 a). The flux scales as $E_{L}{ }^{1.7}$ which is similar to the experimental results. Differences between the modelling and experimental results will arise as GEANT4 does not take into account collective effects of the electrons inside the target or the heating of the bulk target material itself.

The dose per incident joule of laser energy from similar laser-solid interactions has been thoroughly modelled by Fontaine et al [7], as shown in Figure 2 b) with comparison to experimental measurements. This appears to scale as approximately intensity to the power of 0.6 for a fixed wavelength, which slightly weaker but similar to the experimental results shown here if they are divided by the incident laser energy.

\section{X-ray Radiography}

The $\mathrm{x}$-rays were used to investigate a number of objects. An example of an $\mathrm{x}$-ray radiograph is shown in Figure 3 which shows images of a tungsten resolution test grid a) and steel pellets inside concrete $b$ ).

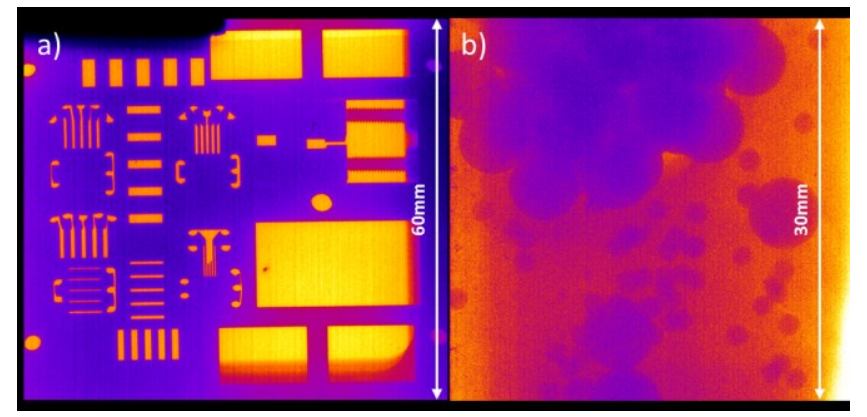

Figure 3: a) A radiograph of a tungsten resolution test grid and b) steel pellets inside concrete.

\section{References}

[1] Courtois, C., "Characterisation of a MeV Bremsstrahlung x-ray source produced from a high intensity laser for high areal density object radiography," Phys. Plasmas 20, 083114 (2013)

[2] Brenner, C.M., "Laser-driven x-ray and neutron source development for industrial applications of plasma accelerators," Plasma Phys. Control. Fusion 58014039 (9pp) (2016)

[3] Clarke, R.J., "Radiological characterisation of photon radiation from ultra-high-intensity laser-plasma and nuclear interactions," J. Radiol. Prot. 26 277-286 (2006)

[4] S. Agostinelli., "Geant4 - a simulation toolkit," Nuclear Instruments and Methods in Physics Research A 506 250-303 (2003)

[5] Danson. C, " Vulcan petawatt: Design, operation and interactions at 5x1020 Wcm-2," Laser and Particle Beams, 23, 87-93, (2005)

[6] Beg, F. N.., "A study of picosecond laser-solid interactions up to 1019 Wcm-2," Phys. Plasmas, Vol. 4, No. 2, (1997)

[7] Compant La Fontaine, A., "Photon dose produced by a high-intensity laser on a solid target," J. Phys. D: Appl. Phys. 47 325201 (18pp)

(2014) 